\title{
Dual immobilization and magnetic manipulation of magnetic nanoparticles
}

\author{
S.Y. Yang ${ }^{\mathrm{a}, 1}$, Z.F. Jian ${ }^{\text {a }}$, H.E. Horng ${ }^{\mathrm{a}, *}$, Chin-Yih Hong ${ }^{\mathrm{b}, *}$, H.C. Yang ${ }^{\mathrm{c}}$, C.C Wu ${ }^{\mathrm{d}}$, Y.H. Lee ${ }^{\mathrm{e}}$ \\ a Institute of Electro-optical Science and Technology, National Taiwan Normal University, Taipei 116, Taiwan \\ ${ }^{\mathrm{b}}$ Department of Mechanical Engineering, Nan-Kai Institute of Technology, Nantou County, Taiwan \\ ${ }^{\mathrm{c}}$ Department of Physics, National Taiwan University, Taipei 106, Taiwan \\ ${ }^{\mathrm{d}}$ Departments of Primary Care and Internal Medicine, College of Medicine, National Taiwan University, Taipei 102, Taiwan \\ e Medical Metrology Center, Korea Research Institute of Standards and Science, Daejeon 305-340, Republic of Korea
}

\section{A R T I C L E I N F O}

\section{Article history:}

Received 24 March 2008

Received in revised form

1 May 2008

Available online 11 June 2008

\section{PACS:}

75.50.Mm

81.20.-n

Keywords:

Magnetic nanoparticles

Bio-vector

Stem cell

\begin{abstract}
A B S T R A C T
By suitably bio-functionalizing the surfaces, magnetic nanoparticles are able to bind specific biomolecules, and may serve as vectors for delivering bio-entities to target tissues. In this work, the synthesis of bio-functionalized magnetic nanoparticles with two kinds of bio-probes is developed. Here, the stem cell is selected as a to-be-delivered bio-entity and infarcted myocardium is the target issue. Thus, cluster designation-34 (CD-34) on stem cell and creatine kinase-MB (CK-MB) (or troponin I) on infarcted myocardium are the specific biomolecules to be bound with bio-functionalized magnetic nanoparticles. In addition to demonstrating the co-coating of two kinds of bio-probes on a magnetic nanoparticle, the feasibility of manipulation on bio-functionalized magnetic nanoparticles by external magnetic fields is investigated.
\end{abstract}

(c) 2008 Published by Elsevier B.V.

\section{Introduction}

By bio-functionalizing the surfaces, magnetic nanoparticles own the affinity with specific biomolecules; furthermore, can be applied to aspects of biology and bio-medicine. For example, by coating antibodies onto magnetic nanoparticles [1,2], magnetic nanoparticles serve as bio-markers for interested tissues or molecules. Magnetic-nanoparticle bio-markers are now popularly used in magnetic resonance imaging [3,4], immunoassay [5-8], DNA assay [9-11], and cell sorting. In addition to bio-markers, biofunctionalized magnetic nanoparticles can be vectors, which are useful in gene transfection and drug delivery $[12,13]$. In the case of bio-markers, it is usual to coat one kind of monoclonal antibodies onto magnetic nanoparticles to achieve a high-specificity labeling on the conjugated biomolecules. However, for the applications of vectors, magnetic nanoparticles prefer to have two kinds of linking epitopes. Thus, magnetic nanoparticles can bind to-bedelivered units via one of the linking epitopes, and specifically anchor at target units via the other linking epitope. In this work,

\footnotetext{
* Corresponding authors.

E-mail addresses: phyfv001@scc.ntnu.edu.tw (H.E. Horng), cyhong@nkc.edu.tw (C.-Y. Hong).

${ }^{1}$ Also with: MagQu Co., Ltd., Sijhih City, Taipei County 221, Taiwan.
}

we would like to concentrate on the topic of magneticnanoparticle vectors.

In the research field of bio-delivery, recently stem cell delivery has attracted much attention due to the promise of stem-cell therapy, in which stem cells are transplanted to a specific region to locally treat failed tissues. For instance, when one suffers from myocardium infarction, regional myocardium of, instead of all over, the heart fails. It is possible to treat the regionally failed myocardium by transplanting stem cells at the failed myocardium. The conventional way to identify the infarcted myocardium is to slice tissues with in-vivo assays. This implies an examination is necessary to localize infarcted myocardium. Then, surgical procedure to plant stem cells at the detected infarcted myocardium. These tedious diagnosis and treatment methods greatly reduce the feasibility of stem-cell therapy in clinical use, and motivate people to search for alternative ways. One promising way is to utilize magnetic nanoparticles to deliver stem cells to infarcted myocardium. In this case, stem cells are bound to magnetic nanoparticles via certain kinds of linking epitopes, and are injected into the treated entity. Through magnetic attraction or natural perfusion, the complexes of stem-cell-magneticnanoparticle are guided to the heart, followed by the residence of the complexes at the infarcted myocardium via another kind of linking epitope on the magnetic nanoparticles. It is obvious that the magnetic-nanoparticle-assisted stem cell delivery is much easier and safer than the conventional way. More efforts have 
a

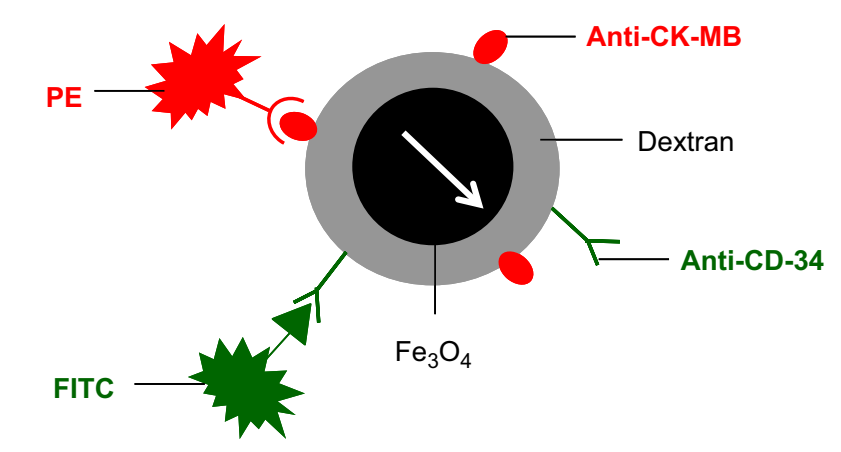

b

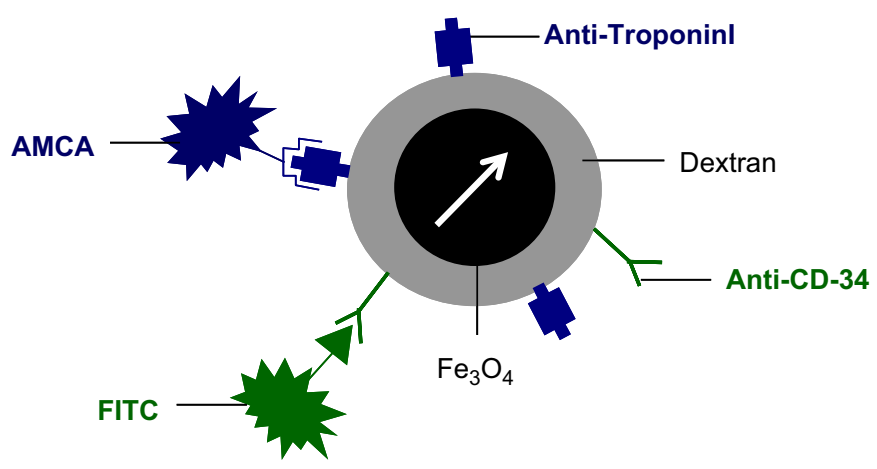

Fig. 1. Schematic pictures of (a) Type I and (b) Type II vectors. The antibodies on Type I vector are anti-CD-34 and anti-CK-MB. The fluorescent markers for these two antibodies are FITC and PE, respectively. The antibodies on Type II vector are anti-CD-34 and anti-troponin I. The fluorescent markers for these two antibodies are FITC and AMCA, respectively.

been employed to develop magnetic-nanoparticle-assisted stemcell therapy from aspects of materials, delivery skills, and detection methods. In this work, we focus on the synthesis of magnetic nanoparticles for stem cell delivery to the infarcted myocardium.

To do this, we coated two kinds of bio-probes for the association with stem cells and infarcted myocardium, respectively. For the specific association between magnetic nanoparticles and stem cells, cluster designation-34 (CD-34) on stem cells is selected as a linking target. Thus, anti-CD-34 is coated onto magnetic nanoparticles for binding stem cells via the affinity of anti-CD-34 on magnetic nanoparticles to CD-34 on stem cells. To achieve the specific association of magnetic nanoparticles with the infarcted myocardium, creatine kinase-MB (CK-MB) and troponin I, which are expressed by infarcted myocardium, are selected as linking targets. Hence, anti-CK-MB or anti-troponin I is coated onto magnetic nanoparticles. Therefore, magnetic nanoparticles coated with anti-CD-34 and anti-CK-MB (or anti-CD-34 and anti-troponin I) can serve as vectors to deliver stem cells to the infarcted myocardium. Hereafter, the magnetic nanoparticles coated with anti-CD-34 and anti-CK-MB are referred to as Type I vector, whereas magnetic nanoparticles coated with anti-CD-34 and anti-troponin I are denoted as Type II vector, as schematically illustrated in Fig. 1(a) and (b), respectively.

\section{Experimental details}

The coating process of antibodies onto magnetic nanoparticles is illustrated in Fig. 2. The magnetic nanoparticles used here are

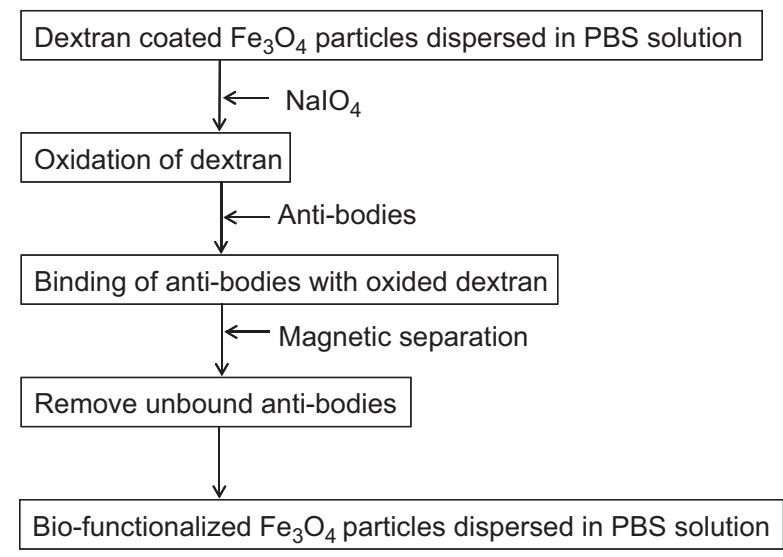

Fig. 2. Flowchart of the coating of antibodies onto magnetic nanoparticles.

dextran-coated $\mathrm{Fe}_{3} \mathrm{O}_{4}$ particles shown in Fig. 1 dispersed in phosphate-buffered saline (PBS) solution (MagQu Co.). The mean diameter of the particles is $58 \mathrm{~nm}$. The dextran serves as a surfactant to achieve the dispersion of magnetic nanoparticles in PBS solution, as well as acts as a linker between $\mathrm{Fe}_{3} \mathrm{O}_{4}$ particles and antibodies. To make antibodies bind to the dextran on the outermost shell of magnetic nanoparticles, $\mathrm{NaIO}_{4}$ solution is added into the magnetic solution to oxidize dexrtan to create aldehyde groups (-CHO). Then, dextran can react with antibodies via the linking of- $\mathrm{CH}=\mathrm{N}-$. Thus, the antibody is bound to dextran at the $\mathrm{F}_{\mathrm{c}}$ part, and the binding between antibody and dextran is covalent and irreversible. Through magnetic separation, unbound antibodies were separated from the solution. For the Type I vector, the antibodies are a mixture of anti-CD-34 and antiCK-MB. Here, $1 \mu \mathrm{g}$ anti-rat-CD-34 (Abcam) and $1 \mu \mathrm{g}$ anti-rabbitCK-MB (Abcam) are solved in $1 \mathrm{ml}$ solution. On the other hand, $1 \mu \mathrm{g}$ anti-rat-CD-34 (Abcam) and $1 \mu \mathrm{g}$ anti-mouse-troponin I (Abcam) are solved in $1 \mathrm{ml}$ solution for synthesizing the Type II vector.

To investigate the co-coating of the two kinds of antibodies on a magnetic nanoparticle, two types of fluorescent bio-markers are added to the magnetic vectors to label these two kinds of antibodies, respectively. For labeling anti-rat-CD-34 and antirabbit-CK-MB of Type I vector, goat-anti-rat-FITC antibody (Abcam) and goat-anti-rabbit-PE antibody (Abcam) are used, where FITC and PE are named after flourescein isothiocyanate and phycoerythrin, respectively. Goat-anti-rat-FITC antibody and goat-anti-mouse-AMCA antibody are used as fluorescent markers for anti-rat-CD-34 and anti-mouse-troponin I, respectively, of the Type II vector, where AMCA is named after aminomethylcoumarin. After the incubation of complexes formed with the fluorescent bio-markers and the antibodies on magnetic nanoparticles, unbound fluorescent bio-markers are removed from the magnetic vector solutions via magnetic separation.

Goat-anti-rat-FITC antibody used here can specifically bind to anti-rat-CD-34, while goat-anti-rabbit-PE specifically associates with anti-rabbit-CK-MB. Furthermore, goat-anti-mouse-ACMA can specifically attach to anti-mouse-troponin I. The fluorescent marker FITC absorbs $495 \mathrm{~nm}$ wavelength ultraviolet light and then emits $528 \mathrm{~nm}$ wavelength green light. Another fluorescent marker PE absorbs $488 \mathrm{~nm}$ wavelength ultraviolet light and then emits $575 \mathrm{~nm}$ wavelength orange-red light. The fluorescent marker AMCA absorbs $350 \mathrm{~nm}$ wavelength ultraviolet light and then emits $450 \mathrm{~nm}$ wavelength blue light. The fluorescent markers and their characterizations to each kind of antibodies on magnetic nanoparticles are listed in Table 1 . Thus, the positions of magnetic nanoparticles coated with anti-rat-CD-34, anti-rabbit-CK-MB, or 
Table 1

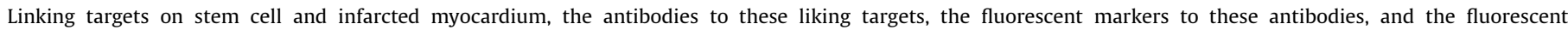
characterizations of these fluorescent markers

\begin{tabular}{|c|c|c|c|}
\hline Cell/tissue & Stem cell & Infarcted myocardium & \\
\hline Linking target & CD-34 & CK-MB & Troponin I \\
\hline Antibody & Anti-rat-CD-34 & Anti-rabbit-CK-MB & Anti-mouse-troponin I \\
\hline Fluorescent marker & FITC & $\mathrm{PE}$ & AMCA \\
\hline Absorption wavelength (nm) & 495 & 488 & 350 \\
\hline Emitting wavelength (nm) & 528 (green) & 575 (orange-red) & 450 (blue) \\
\hline
\end{tabular}

The antibodies are coated on magnetic nanoparticles.
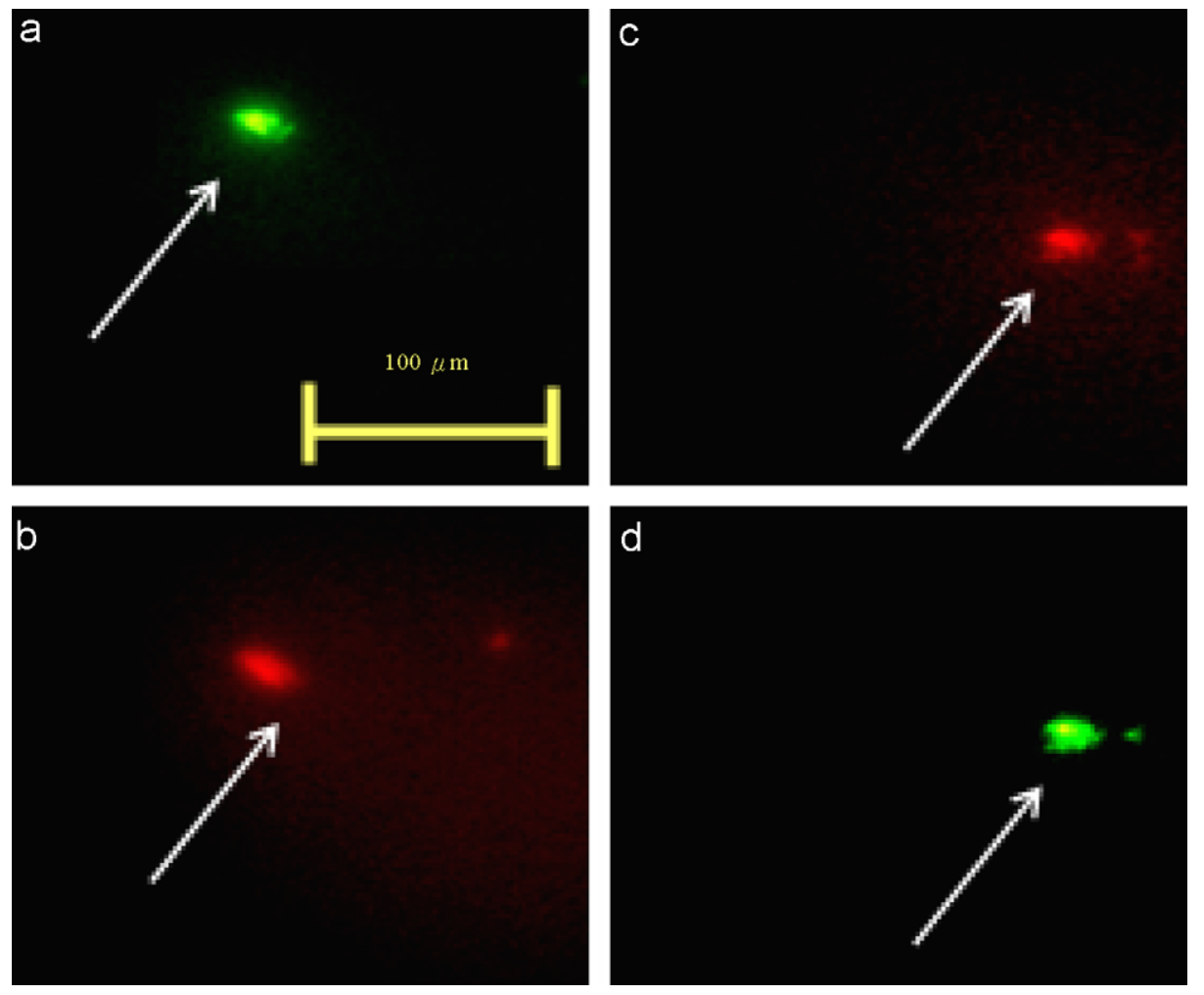

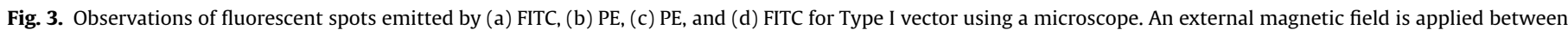
observations (b) and (c).

anti-mouse-troponin I can be identified with green, red, or blue spot lights by using a fluorescent microscope.

\section{Results and discussion}

Fig. 3(a) shows the image of diluted Type I vector using a fluorescent microscope with a green-light band-pass filter $(500-540 \mathrm{~nm})$. As indicated with the arrow, a green spot was observed. This green spot is generated with FITC. Then, the bandpass filter of the microscope is switched to a red-light band-pass filter (570-625 nm). A red spot shown in Fig. 3(b) was found to be exactly at the same position as the green spot observed in Fig. 3(a). Since the red spot is emitted with PE, the coincidence of the green spot and the red spot evidences that goat-anti-rat-FITC and goat-anti-rabbit-PE are mixed at the same complex. In order to verify that this complex is on a magnetic nanoparticle, a magnet is put beside the sample to move the magnetic nanoparticles via magnetic attraction. In this case, the red spot observed in Fig. 3(b) was expected to move if goat-anti-rabbit-PE was bound to the magnetic nanoparticle. The movement of the red spot towards the magnet was confirmed, as illustrated in Fig. 3(c). The red spot in Fig. 3(c) locates at a different position from that in Fig. 3(b). This result definitely reveals that goat-antirabbit-PE is on the magnetic nanoparticle. Furthermore, the translation of the red spot in Fig. 3(b) and (c) evidences the feasibility of manipulation of the bio-functionalized magnetic nanoparticles by external magnetic fields. Then, the band-pass filter of the microscope is switched back to green-light band-pass filter to find the green spot shown in Fig. 3(a). It was observed in Fig. 3(d) that the green spot now locates coincidently with the red spot shown in Fig. 3(c). By comparing the images in Fig. 3(a) and (d), the green spot follows the same movement as that of the red spot in Fig. 3(b) and (c). Hence, it can be concluded that goatanti-rat-FITC and goat-anti-rabbit-PE associate with the same magnetic nanoparticle. Since the linking between goat-anti-ratFITC/goat-anti-rabbit-PE and magnetic nanoparticle is anti-ratCD-34/anti-rabbit-CK-MB, the results shown in Fig. 3(a) reveal the co-coating of anti-rat-CD-34 and anti-rabbit-CK-MB on a magnetic nanoparticle.

The co-coating of anti-mouse-troponin I and anti-rat-CD-34 for the Type II vector is examined. Fig. 4(a) shows the image taken 


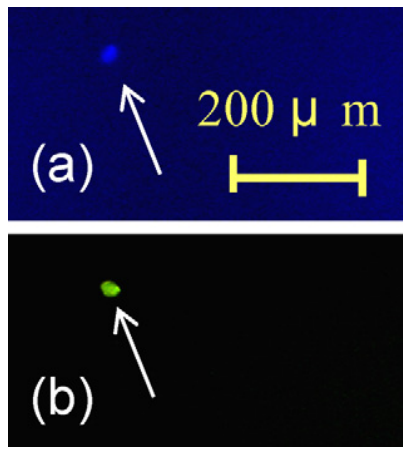

Fig. 4. Observations of fluorescent spots emitted by (a) FITC and (b) ACMA for Type II vector using a microscope.

with the microscope equipped with a blue-light band-pass filter (400-600 nm). A blue spot was observed in the upper-left area, as indicated with an arrow. The blue spot is generated by AMCA on the goat-anti-mouse-AMCA (Upstate). When the band-pass filter of the microscope is changed to a green-light band-pass filter, the image is shown in Fig. 4(b). Clearly, the green spot, which is generated with goat-anti-rat-FITC, coincides with the blue spot shown in Fig. 4(a). These results imply that anti-mouse-troponin I and anti-rat-CD-34 reside at the same magnetic nanoparticle.

\section{Conclusion}

Through the synthetic processes developed in this work, more than one kind of antibody or bio-probes can be simultaneously coated onto a magnetic nanoparticle. So that, such magnetic nanoparticles are promisingly used for bio-vectors for specific bio-entity (e.g. stem cell, here) to target tissues (e.g. infarcted myocardium). The results also show the feasibility of manipulation of such magnetic bio-vectors by using external magnetic fields.

\section{Acknowledgment}

This work is supported by National Science Council, Taiwan under Grant nos., NSC95-2120-M-003-001, 96-2314-B-002150-MY3, 96-2628-M-003-002, 95-2112-M-003-017-MY2, and 96-2752-M002-016-PAE.

\section{References}

[1] Wanquan Jiang, H.C. Yang, S.Y. Yang, H.E. Horng, J.C. Hung, Y.C. Chen, Chin-Yih Hong, J. Magn. Magn. Mater. 283 (2004) 210.

[2] H.E. Horng, S.Y. Yang, Y.W. Huang, W.Q. Jiang, C.-Y. Hong, H.C. Yang, IEEE. Trans. Appl. Supercond. 15 (2005) 668.

[3] D. Roberts, W.L. Zhu, C.M. Frommen, Z. Rosenzweig, J. Appl. Phys. 87 (2000) 6208.

[4] D.E. Sosnovik, E.A. Schellenberger, M. Nahrendorf, M.S. Novikov, T. Matsui, Ge. Dai, F. Reynolds, L. Grazette, A. Rosenzweig, R. Weissleder, L. Josephson, Magn. Reson. Med. 54 (2005) 718.

[5] R. Kötitz, W. Weitschies, L. Trahms, W. Brewer, W. Semmler, J. Magn. Magn. Mater. 194 (1999) 62.

[6] S.K. Lee, W.R. Myers, H.L. Grossman, H.-M. Cho, Y.R. Chemla, J. Clarke, Appl. Phys. Lett. 81 (2002) 3094.

[7] K. Enpuku, T. Minotani, T. Gima, Y. Kuroki, Y. Itoh, M. Yamashita, Y. Katakura, S. Kuhara, Jpn. J. Appl. Phys. 38 (1999) L1102.

[8] H.C. Yang, S.Y. Yang, S.H. Liao, G.L. Fang, W.H. Huang, C.H. Liu, H.E. Horng, C.-Y. Hong, J. Appl. Phys. 99 (2006) 124701.

[9] S. Katsura, T. Yasuda, K. Hirano, A. Mizuno, S. Tanaka, Supercond. Sci. Technol. 14 (2001) 1131.

[10] Y. Ichiyanagi, S. Moritake, S. Taira, M. Setou, J. Magn. Magn. Mater. 310 (2007) 2877.

[11] M. Pita, J.M. Abad, C. Vaz-Dominguez, C. Briones, E. Mateo-Martí, J.A. MartínGago, M. del P. Morales, V.M. Fernández, J. Colloid Interface Sci. 321 (2008) 484.

[12] O. Mykhaylyk, D. Vlaskou, N. Tresilwised, P. Pithayanukul, W. Möller, C. Plank, J. Magn. Magn. Mater. 311 (2007) 275.

[13] S.Y. Yang, J.S. Sun, C.H. Liu, Y.H. Tsuang, L.T. Chen, C.-Y. Hong, H.C. Yang, H.E. Horng, Artif. Organs 32 (2008) 195. 